solution gives all surfaces of constant mean curvature can be reduced to a simple form. By a suitable choice of parameters we have $D=D^{\prime \prime}=1$, and there is no loss in assuming that the square of the mean curvature is unity. When these values are substituted in the Gauss equation,* it reduces to

$$
\frac{1}{F} \frac{\partial^{2} \log F}{\partial u \partial v}=\frac{1}{F^{2}}-1 \text {. }
$$

If we put $\log F=\theta$, this equation becomes

$$
\frac{\partial^{2} \theta}{\partial u \partial v}=e^{-\theta}-e^{\theta} .
$$

When a solution of this equation is known, all of the fundamental coefficients of the surface are given.

Princeton, N. J.,

September 20, 1902.

\title{
SUPPLEMENTARY NOTE ON THE CALCULUS OF VARIATIONS.
}

BY DR. E. R. HEDRICK.

IN a recent paper in the BuLletin, $\dagger$ the present writer stated the theorem: "The condition $\partial^{2} f / \partial y^{\prime 2}>0$ for all $x, y$ on the curve $C$, and for all $y^{\prime}$ considered, is a sufficient condition for a minimum" (of the integral $\int f\left(x, y, y^{\prime}\right) d x$ along the curve $C$ ), provided that certain preliminary requirements are satisfied. This theorem stands in apparent contradiction with an example given by Professor Bolza ; $\ddagger$ and while the contradiction is only apparent, it seems fitting to point out clearly the actual agreement of my results with those of Professor Bolza.

In the article mentioned I have required (page 11) that the integral $\int f\left(x, y, y^{\prime}\right) d x$, shall be considered only in a region $R$ (of the $x, y, y^{\prime}$ space), such that the integrand $f\left(x, y, y^{\prime}\right)$ is

* Bianchi, l. c., p. 67.

† "On the sufficient conditions in the calculus of variations," BuLletin, vol. 9 (2), no. 1 (Oct., 1902), p. 15.

$\ddagger$ "Some instructive examples in the calculus of variations," BULLETIN, vol. 9 (2), no. 1 (Oct., 1902), Example II, p. 9. 
analytic at each point of $R, i$. e., for all sets of values of $x, y$, $y^{\prime}$ in $R$. A region is here a closed manifold of points, $i$. e., it possesses a boundary, so that a point upon the boundary of $R$ belongs to the region. Hence for the reasoning throughout it is necessary to choose such a region $R$. It is true that this restriction is by no means necessary for most of the results of the paper, but without it some of the proofs, and in particular the above theorem, would have to be modified. It is to be noticed, however, that we may choose an unbounded region $R_{1}$ (i. e., exclusive of the boundary) provided $R_{1}$ lies wholly within a region $R$ such as is required above, and that all the results of the paper hold when the given integral is considered only for points inside of $R_{1}$, or in particular inside of $R$ itself. In other words, the results given hold in any region (inclusive or exclusive of the boundary) provided the points for which the integrand $f\left(x, y, y^{\prime}\right)$ is not analytic be excluded from the region (i. e., from its interior and from its boundary).

In Professor Bolza's example, I would then, for the purposes of my paper, first choose as my region $R$ the region

$$
R: \quad|x| \leqq A ; \quad|y| \leqq A ; \quad\left|y^{\prime}\right| \leqq A .
$$

Any positive number $A$ having been chosen for the example once for all, the investigation of minima depends on our ability to find, secondly, a value for $\delta$ so small that the supposed solution renders the integral value less than along any other curve for which

$$
|\eta(x)|<\delta, \quad x_{0} \leqq x \leqq x_{1} .
$$

It is seen at once that any order of choice of these quantities $A$ and $\delta$ corresponds exactly to the same order of choice of the $h$ and $k$ of Professor Bolza's article, respectively; whereas the order of choice necessary for the considerations of my paper is exactly opposite to that made by Professor Bolza. For, from my standpoint, $k$ cannot be first chosen and then kept fixed while $h$ (secondly) approaches zero, as is necessary for Professor Bolza's argument. A choice of $h$ after $k$ has been chosen and fixed, necessitates a choice of $A \geqq k / h$. This I would regard as the first step, after which I would have $\delta$ at my disposal, which would necessitate a re-choice of $k$, if $\delta$ be chosen less than $k$. It readily follows that, from my standpoint, the conclusion $\Delta I<0$ cannot be drawn, and that the $X$ axis actually renders 
the integral in question a strong minimum,* in the above region $R, i . e$. , in any region of values of $x, y, y^{\prime}$ from the interior and boundary of which the values $x=\infty, y=\infty, y^{\prime}=\infty$ are excluded. Accordingly the example cited, as also Professor Bolza's theorem at the top of page 9, are perfectly reconcilable with my theorem quoted above.

The integrand in question is discontinuous for infinite values of either $x$ or $y$ or $y^{\prime}$, and in fact becomes infinite to the second order for $y^{\prime}=\infty$. It may be pointed out that the same apparent contradiction will arise in the case of any integral whose integrand becomes infinite to the second order for any value of $y^{\prime}$ (not necessarily $y^{\prime}=\infty$ ); but again the contradiction is only apparent. It might be questioned whether simple ordinary integrals, such as the integral of length, $\int \sqrt{1+y^{\prime 2}} d x$, do not present similar phenomena, so that the results of my paper would be restricted in their application, in all ordinary examples, to a closed region (of the $x, y, y^{\prime}$ space) in which the integrand is analytic. Such are indeed the formal requirements of the paper. But it may be noted that if the transformed integral

$$
\int f\left(x, y, \frac{1}{x^{\prime}}\right) x^{\prime} d y
$$

where $x^{\prime} \equiv d x / d y$, be analytic for the directions previously excluded $\left(y^{\prime}=a, x^{\prime}=1 / a\right)$, then the whole reasoning applies to the comparison curves previously excluded, by a simple process of transformation and subdivision of the curve, at least provided that the comparison curves in question satisfy the simple requirement of not being parallel to each axis an infinite number of times in any interval.

And further, the requirement made on page 11 is by no means necessary for the proof of most of the theorems. In view, however, of the aims of the article, it was deemed advisable to include this restriction, in order to simplify the proofs, and in order to arrive at the theorem mentioned above without too extended a discussion of possible alternate restrictions.

Sheffield Scientific School, E. R. HEDRICK. November, 1902.

* The distinction between strong and weak minima holds as usual because the slope restriction $\left|y^{\prime}\right| \leqq A$ was made before any particular extremal was found, and does not regard any particular extremal. 